\title{
Active Disturbance Rejection Control of Carrier-based Aircraft Based on Multi-objective Immune Optimization
}

\author{
CHEN Junfeng $^{a}$, HAN Wei ${ }^{b}$, SU Xichao ${ }^{c}$ \\ Department of Airborne Vehicle Engineering, Naval Aeronautic Engineer Institute, Yantai 264001, \\ China

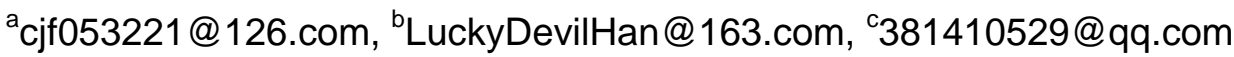

Keywords: ADRC; multi-objective optimization; improved Memetic immune algorithm

\begin{abstract}
In the design of control system, if only the control precision is taken into account, the control energy may be too large, but in a real system, control energy is often limited. Based on this, the attack angle error and engine power are treated as optimization objectives, then the parameters optimization of ADRC are viewed as multi-objective optimization. The parameters of active disturbance rejection control(ADRC) are calculated by improved Memetic immune algorithm, the Pareto set of attack angle error and engine power are obtained. The results show that the engine power could be reduced effectively within the range of allowable attack angle error by choosing parameters reasonably in Pareto set.
\end{abstract}

\section{Introduction}

When modern carrier-based aircraft landing, the attack angle need to be constant mostly ${ }^{[1]}$, that is, on the premise of attitude stabilization, the attack angle is controlled by engine thrust, so as to achieve flight path angle following command fast and accurately ${ }^{[2][3]}$.

The Active Disturbance Rejection Control(ADRC) ${ }^{[4]}$ can estimate and compensate the disturbance in real time, it is a robust control strategy based on "error elimination". Since the ADRC was proposed, it has been used in the field of aviation and aerospace for a considerable number of applications [5][6][7]. But because of its amounts of adjustable parameters, it is needed to be optimized based on different parameters tuning principles or optimization algorithms ${ }^{[8][9][10][11]}$.

The existing optimization strategies for the parameters tuning of the ADRC are only based on the control accuracy, which may cause large energy consumption, so the actual energy limitation may cause control performance degradation, or perhaps fewer energy was needed to get almost the same control accuracy. In view of this, the angle of attack error and the thrust were viewed as two separate optimization objectives, and the improved Memetic immune algorithm was used to optimize the parameters of the ADRC.

\section{Problem Description}

The ADRC generally includes tacking differentiator(TD) ,which is used to arranging the transient process, the extended state observer (ESO), which is used to observe the disturbance,and the nonlinear state error feedback (NLSEF).

The traditional method for the optimization of the parameters of ADRC is based on some criteria, and the absolute value of the error combined with time is the only optimization objective. Since the ADRC does not take the upper limitation of the control energy into account, the controller parameters tuned by traditional method may cause the control energy exceed the energy limitation.

Therefore, this paper take the control energy as another object to be optimized, so the optimization problem of the parameters of the ADRC can be described as:

$$
\left\{\begin{array}{l}
\min f_{1}=\int t|e| \mathrm{d} t \\
\min f_{2}=\int|u| \mathrm{d} t
\end{array}\right.
$$

Combined (1), (2) and the relevant parameters' selection principle, the search space can be 
determined as: $\beta_{\alpha 1} \in[0,1000], \beta_{\alpha 2} \in[0,5000], \alpha_{\alpha} \in(0,1), \Delta_{\alpha} \in[0.001,1), b_{0} \in(0,10], c_{\alpha} \in(0,5], r_{\alpha} \in(0,50]$, $h_{\alpha} \in[0.001,1)$.

\section{Improved Memetic Immune Optimization Algorithm (IMIAMO)}

Memetic immune optimization algorithm (MIAMO) is developed by Qi Yutao ${ }^{[12]}$, which is based on NNIA ${ }^{[13]}$. and NNIA-II ${ }^{[14]}$. The MIAMO is more efficiency, the basic steps can refer to [12].

However, in solving the classical multi-objective test problems such as ZDT6 and DTLZ2, the MIAMO algorithm is easy to fall into local optimum. In order to improve the search efficiency of and the approximation ability to the global optimal solution, the MIAMO is improved as:

(1) the Memetic operator is improved, and the new generation of antibodies was non-uniform mutated $^{[15][16]}$ after differential evolution, to improve the global optimal approximation ability;

(2) the crossover mutation operator in the original algorithm is improved to new operator, which is based on the rank ${ }^{[17]}$.

\section{Algorithm Verification}

In order to test the performance of the improved algorithm, two standard multi-objective optimization test problems ${ }^{[18][19][20]}$ are selected: ZDT6 and DTLZ2. The experimental results of the MIAMO and NSGA-II algorithms are compared.

The performance of the algorithm is evaluated by using a comprehensive evaluation index (IGD) ${ }^{[21]}$. IGD could measure the convergence and diversity of multi-objective optimization algorithm, the smaller the value, the better performance of the algorithm ${ }^{[12]}$. In order to verify the robustness of the algorithm, the test problems are calculated 30 times using the different algorithms respectively. The IGD value of the 30 calculation results is used as the final evaluation index.

The algorithm is set up as follows: the crossover mutation operator is set with the same parameters, the crossover probability is 0.8 , the mutation probability is $0.8+1 / n, n$ is search space dimension, and the cross distribution index and the variation distribution index are set to 20 .

In IMIAMO and MIAMO algorithms, the size of the non-dominated population $\mathrm{nD}$ is set to 100 , the size of the activation population $\mathrm{nA}$ is set to 20 , the size of the clone population $\mathrm{nC}$ is set to 50 , the neighbor list $S$ is set to 20 , and the PD is set to 0.5 . The population size of the NSGA-II algorithm is set to 100 . The three algorithms are set to iterate 200 times. The results are shown in figure 1- 5.
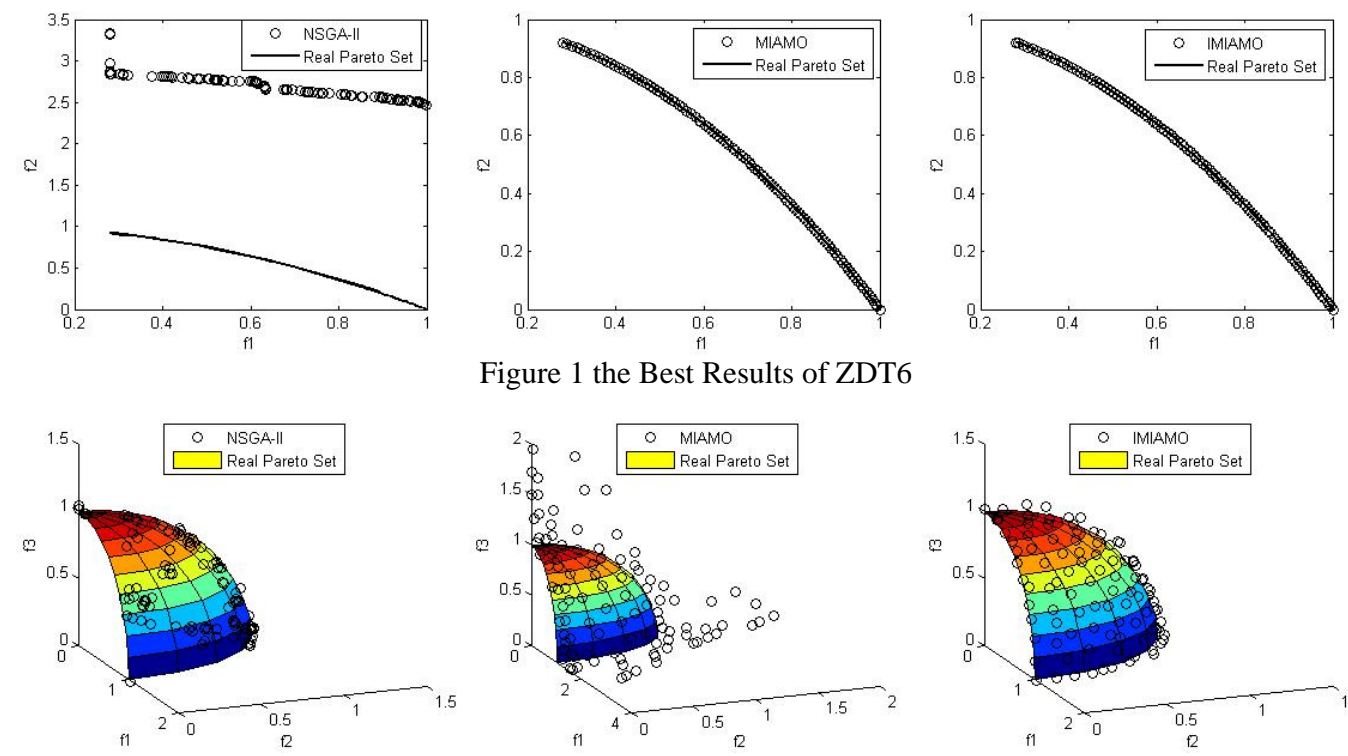

Figure 1 the Best Results of ZDT6
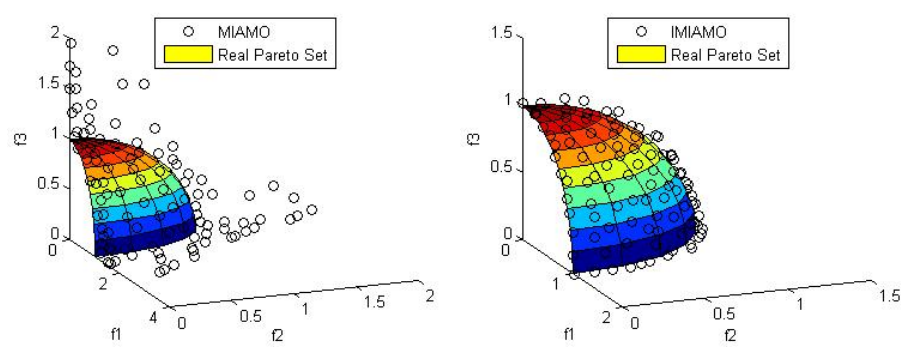

Figure 2 the Best Results of DTLZ2 

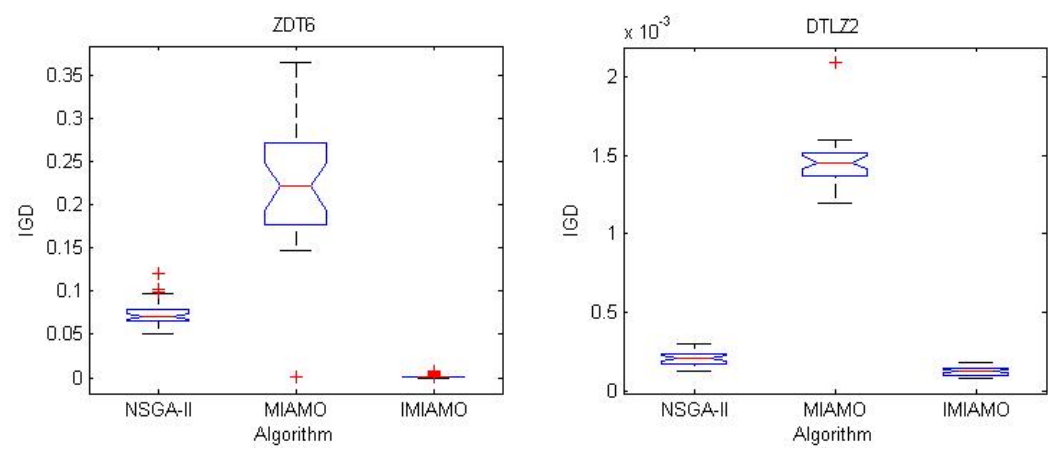

Figure 3the IGD of Three Different Algorithms

Figure 1 are the best results of the three algorithms for the test problem ZDT6. It can be seen that the NSGA-II do not obtain the Pareto set in all the 30 evolutions; however MIAMO can obtain the Pareto set, but most of them fall into the local optimal solution.

Figure 2 are the best results of the three algorithms for the test problem DTLZ2. By the results we can see that the convergence of DTLZ2 is better, but the diversity is poor. MIAMO is unable to converge to the true Pareto set, but IMIAMO obtained the Pareto set with a good diversity.

Figure 3 is IGD value of three different algorithms. Although ZDT6 can converge to the real Pareto set, but the robustness of the algorithm is poor, the optimal results in the case of the MIAMO are treated as a wild value, and the median is greater than NSGA-II and IMIAMO.

From the above graphs, the MIAMO is better than NSGA-II or IMIAMO in solving the problem of solving the two dimensional multi-objective optimization. The diversity of the solution is better than that of NSGA-II when solving the problem of 3D multi-objective optimization. And MIAMO cannot guarantee the convergence to the true Pareto optimal solution set in the solution of 3D multi-objective optimization problem. In conclusion, the IMIAMO algorithm is better than MIAMO algorithm in solving some multi-objective optimization problems.

\section{Optimization Results of ADRC}

Taking (3) as the optimization objective, the parameters of ADRC are optimized by IMIAMO algorithm, and the Pareto solution set of attack angle error and engine thrust is shown in Figure 4.

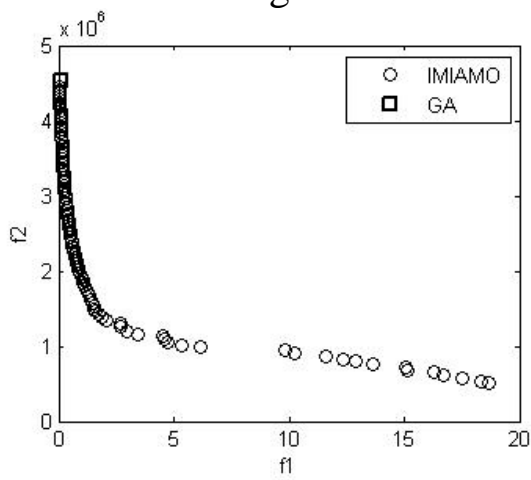

Figure 4 the Pareto Set of Attack Angle Error and Engine Thrust

In figure $4, f_{1}=\int t|e| \mathrm{d} t, f_{2}=\int|u| \mathrm{d} t$. The smaller the angle of attack, the smaller the error of the angle of attack is. In Figure 4, we can see that the results of single objective optimization are included in the results of the Pareto set, the control energy can be reduced efficiently by choosing proper parameters. 

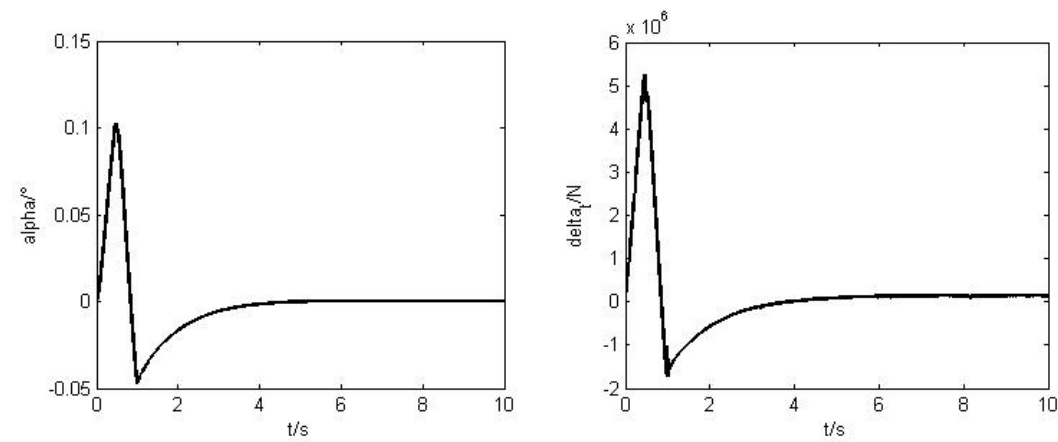

Figure 5 the Attack Angle Error and Engine Thrust Obtained by Single-objective Algorithm
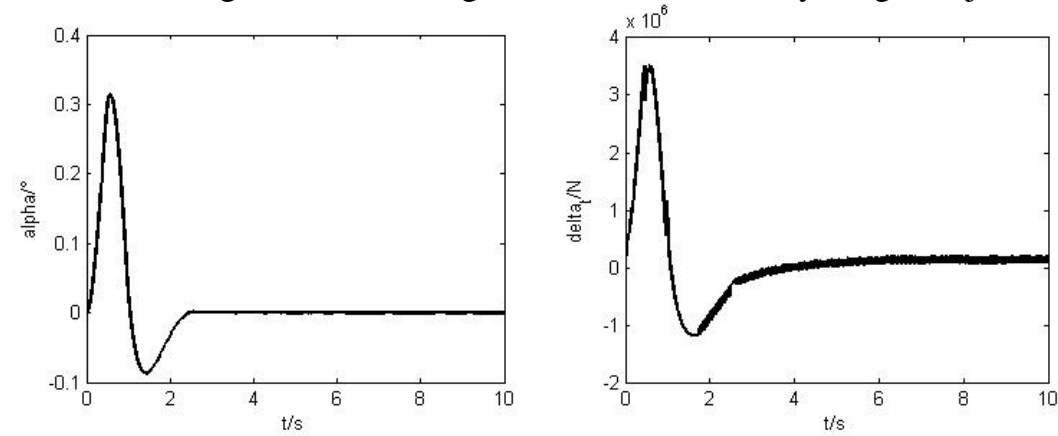

Figure6 the Attack Angle Error and Engine Thrust Obtained by Multi-objective Algorithm

Figure 5 and Figure 6 are single objective and multi-objective optimization results respectively. In the case of almost the same attack angle error, the maximum thrust of the engine is reduced by about $1 / 3$, and the attack angle rate is relatively flat. The results show that the algorithm can effectively reduce the required engine thrust with the reasonable selection of the controller parameters.

\section{Conclusions}

This paper designed the ADRC controller of carrier-based aircraft's power compensation system, the parameters of the controller are tuning by improved MIAMO with the attack angle error and the engine thrust as two different optimal objectives. The MIAMO algorithm is improved to overcome the drawback of falling into the local optima in solving some multi-objective optimization problems, the experimental results verify the validity of the improved algorithm; the Pareto set of attack angle error and engine thrust is obtained by IMIAMO, the control energy can be reduced effectively by choosing parameters rationally.

\section{Reference}

[1]. Yang Yidong. Guidance and Control of Carrier Aircraft Landing[M]. National Defense Industry Press. Beijing, China, 2007.

[2]. Martorella P, Kelly C P, Nastasi R. Rrecision flight path control in carrier landing approach-a case for integrated system design[C]. AIAA paper 81-1710, 1981.

[3]. Fortenbangh R L. Practical integration of direct lift control into an automatic carrier landing system[C]. AIAA paper 72-873, 1972.

[4]. Han Jingqing. Active disturbance rejection control technique — the technique for estimating and compensating the uncertainties[M]. National Defense Industry Press. Beijing, China, 2008.

[5]. Qi Naiming, Qin Changmao, Song Zhiguo. Improved ADRC cascade decoupling controller design of Hypersonic vehicle[J]. Journal of Harbin Institute of Technology, 2011,43(11): 34-38.

[6]. Wu Zhong, Huang Liya, Wei Kongming, et al. Active disturbance rejection control of attitude for spacecraft[J]. Control Theory \& Applications, 2013,30(12):1617-1622.

[7]. Hu Haiyan, Wen Yang, Yang Lingyu, et al. Design of attitude controller based on active disturbance rejection control for structural damaged aircraft[J]. Flight Dynamics, 2013,31(3): 239-243. 
[8]. Li Shuqing, Zhang Shengxiu, Liu Yinan, et al. Parameter-tuning in active disturbance rejection controller using time scale[J]. Control Theory \& Applications, 2012,29(1): 125-129.

[9]. Hu Yi, Wang Mingang, Yang Yao. Parameters Tuning of Active Disturbance Rejection Controller Based on Artificial Fish Swarm Algorithm[J]. Command Control \& Simulation, 2013,35(2): 90-92.

[10]. Wu Lei, Bao Hong, Du Jingli, et al. A Learning Algorithm for Parameters of Automatic Disturbances Rejection Controller[J]. ACTA AUTOMATICA SINICA, 2014,40(3): 556-560.

[11]. Zhou Xuanzheng, Zhang Baoguo. A Study on Parameter Tuning for Active Disturbance Rejection Controllers[J]. ELECTRICAL AUTOMATION, 2014,36(2): 23-24.

[12]. Qi Yutao, Liu Fang, Chang Weiyuan, et al. Memetic Immune Algorithm for Multiobjective Optimization[J]. Journal of Software, 2013,24(7): 1529-1544.

[13]. Gong MG, Jiao LC, Du HF, et al. Multi-Objective immune algorithm with nondominated neighbor-based selection[J]. Evolutionary Computation, 2008,16(2):225-255.

[14]. Yang DD, Jiao LC, Gong MG, et al. Adaptive ranks and K-nearest neighbour list based multiobjective immune algorithm[J]. Computational Intelligence, 2010,26(4): 359-385.

[15]. Jiao Licheng, et al. The theory and application of multi-objective immune optimization algorithm[M]. Science Press, Beijing, China, 2010.

[16]. Michalewicz Z. Genetic Algorithm + Data Structure = Evolution Programs[M]. Berlin: Springer-Verlag, 1992.

[17]. Tao Yang. Research on Modeling of Typical Atmospheric Disturbance Wind Fields and the Landing/Escaping Performances of the Carrier Aircraft[D]. Naval Aeronautical Engineering Institute, Yantai, 2014.

[18]. Deb K, Thiele L, Laumanns $\mathrm{M}$, et al. Scalable multi-objective optimization test problems[C]. Congress on Evolutionary Computation, 2002: 825-830.

[19]. Deb K. Multi-objective genetic algorithms: Problem difficulties and construction of test problem[C]. Evolutionary Computation, 1999,7(3): 205-230.

[20]. Zitzler E, Deb K, Thiele L. Comparison of multi-objective evolutionary algorithm: empirical results[J]. Evol. Comput. (S1063-6560), 2000, 8(2): 173-195.

[21]. Neri F, Cotta C. Memetic algorithms and Memetic computing optimization: A literature review[J]. Swarm and Evolutionary Computation, 2012,2:1-14. 